\title{
ON THE COLOURINGS OF GRAPHS
}

\author{
W. T. Tutte
}

(received March 9, 1961)

A graph $G$ is defined by a set $V(G)$ of vertices, a set $E(G)$ of edges, and a relation of incidence which associates with each edge two distinct vertices called its ends. We consider only the case in which $V(G)$ ard $E(G)$ are both finite.

An $n$-colouring of $G$ is usually defined as a mapping $f$ of $V(G)$ into the set of integers $\{1,2, \ldots, n\}$ which maps the two ends of any edge onto distinct integers. The integers 1 to $\mathrm{n}$ are the $n$ "colours". Much work has been done on n-colourings in recent years, especially by G. A. Dirac.

Let us introduce a complication by assigning to each edge $A$ a colour $g(A)$ and redefining an $n$-colouring of $G$ as a mapping $f$ of $V(G)$ into $\{1,2, \ldots, n\}$ such that the ends of an edge $A$ are not both mapped into $g(A)$. The two ends of an edge can now receive the same colour, provided that it is not that of the edge. The old definition corresponds to the case in which any two vertices joined by an edge are joined by $n$ edges of different colours.

Suppose a vertex $v_{0}$ is joined to vertices $v_{1}, v_{2}, \ldots, v_{n}$ by edges of colours $1,2, \ldots, n$ respectively. Then it is easily seen that there is no $n$-colouring of $G$ in which $v_{1}$ has colour 1, $v_{2}$ has colour 2 and so on. It is found that from the original requirement that the ends of an edge $A$ must not both have the colour $g(A)$ we can deduce a hierarchy of colourpropositions. The typical colour-proposition may be represented by a symbol $\left[S_{1}, S_{2}, \ldots, S_{n}\right]$, where the $S_{i}$ a re subsets of $\mathrm{V}(\mathrm{G})$. It asserts the falsity of the statement that $G$ has an $n$ colouring in which every member of $S_{1}$ has colour 1 , every member of $\mathrm{S}_{2}$ has colour 2, and so on. All colour-propositions in which the $S_{i}$ are not disjoint are true. We call them trivial.

Canad. Math. Bull. vol. 4, no. 2, May 1961. 
The procedure for deducing new colour-propositions from old ones is as follows. Suppose we have n true colour-propositions

$$
\begin{aligned}
& P_{1}=\left[s_{11}, s_{12}, s_{13}, \ldots, s_{1 n}\right] \\
& P_{2}=\left[s_{21}, s_{22}, s_{23}, \ldots, s_{2 n}\right] \\
& \cdot . \cdot \cdot . \cdot . \cdot . . . \cdot \cdot \\
& P_{n}=\left[s_{n 1}, s_{n 2}, s_{n 3}, \ldots, s_{n n}\right]
\end{aligned}
$$

and suppose there is a vertex $\mathrm{v}$ which belongs to each diagonal set $\mathrm{S}_{i i}$. Write

$$
z_{j}=\left(U_{i=1}^{n} s_{i j}\right)-\{v\},(j=1,2, \ldots, n) .
$$

Then $Q=\left[Z_{1}, z_{2}, \ldots, Z_{n}\right]$ is a true colour-proposition. For suppose there is a colouring $C$ in which each member of $Z_{i}$ has colour $i$ (for each i). Then if $f(v)=j$ the colour-proposition $P_{j}$ must be false. We write

$$
Q=\left\{P_{1}, \dot{P}_{2}, \ldots, P_{n}\right\},
$$

and say that $Q$ is the compound of the $P_{i}$ at $v$.

If $S=\left[S_{1}, S_{2}, \ldots, S_{n}\right]$ and $T=\left[T_{1}, T_{2}, \ldots, T_{n}\right]$ are colour-propositions such that $S_{i} \subseteq T_{i}$ for each $i$ we say that $S$ precedes $T$. The truth of $S$ then implies that of $T$. Note that $S$ precedes $S$.

We can construct a hie rarchy of "basic" colour-propositions as follows. Those of rank 1 a re the original colour-propositions, each asserting that the two ends of some edge do not both have a particular colour. For example if an edge $A$ of colour 1 has ends $\mathrm{x}$ and $\mathrm{y}$ the corresponding basic colour-proposition of rank 1 is

$$
[\{x, y\}, \emptyset, \emptyset, \ldots, \emptyset] \text {. }
$$

Basic colour-propositions of ranks 2, 3, .. are defined successively. A colour-proposition is basic and of rank $n$ if it is (i) true, (ii) non-trivial, (iii) a compound of basic colourpropositions of ranks $<n$, and (iv) not preceded by any basic 
colour-proposition of rank < $n$. The process must of course terminate at some finite rank $\lambda(G)$. We denote the resulting hierarchy of basic colour-propositions by $H(G)$.

We are led to enquire whether every true and non-trivial colour-proposition is preceded by a member of $H(G)$. In what follows we answer this question in the affirmative. But first we make a generalization in order to simplify the proof.

We define a chromophore $C$ as a finite set $V$ of vertices for which certain non-trivial colour-propositions $\left[s_{1}, \overline{\left.S_{2}, \ldots, s_{n}\right]}\right.$ are asserted. An $n$-colouring of $C$ is a mapping $f$ of $V$ into $\{1,2, \ldots, n\}$ which is consistent with all the asserted colour-propositions. Any one of the asserted colour-propositions which is not preceded by another is a basic colour-proposition of rank 1. The basic colour-propositions of ranks $2,3, \ldots$, are defined successively as before. We thus obtain a hierarchy $H(C)$. We can say that a graph is a chromophore in which the colour-propositions of rank 1 have a special form.

THEOREM 1. Every true non-trivial colour-proposition of a chromophore $C$ is preceded by a member of $H(C)$.

Proof. If possible let $C$ be a chromophore for which the theorem fails and which has the least number of vertices consistent with this condition.

Let $Q=\left[Q_{1}, Q_{2}, \ldots, Q_{n}\right]$ be any true non-trivial colourproposition of $C$. Suppose first that

$$
V=U_{i=1}^{n} Q_{i}
$$

Then $Q$ is preceded by some basic colour-proposition of rank 1 , for otherwise it would define a colouring of $C$ in which each member of $Q_{i}$ had colour $i$. This would contradict the definition of $Q$.

We may now suppose $C$ has a vertex $v$ belonging to no set $Q_{i}$. We define a chromophore $C^{\prime}$ on $V-\{v\}$. As its initially asserted colour-propositions we take all the basic colourpropositions $\left[P_{1}, P_{2}, \ldots, P_{n}\right]$ of $C$ such that $v$ belongs to no $P_{i}$. We note that $C^{\prime}$ can have no basic colour-proposition of rank > 1 . 
If $Q$ is a colour-proposition of $C^{\prime}$ it is preceded by a basic colour-proposition of $C^{\prime}$ by the definition of $C^{\prime}$, and the refore by a basic colour-proposition of $\mathrm{C}$. We may therefore assume that the re is a colouring $f^{\prime}$ of $C^{\prime}$ such that $f^{\prime}(x)=i$ whenever $x \in Q_{i} \quad(i=1,2, \ldots, n)$. Let $f_{i}$ denote that mapping of $V$ into $\{1,2, \ldots, n\}$ which agrees with $f^{\prime}$ on $\mathrm{V}-\{\mathrm{v}\}$ and satisfies $f_{i}(v)=i,(i=1,2, \ldots, n)$.

Now $f_{i}$ is not a colouring of $C$ since $Q$ is a colourproposition of C. Hence there is a basic colour-proposition $P_{i}=\left[S_{i 1}, S_{i 2}, \ldots, S_{i n}\right]$ of $C$ of rank 1 such that $f_{i}(x)=j$ for each $x \in S_{i j}(j=1,2, \ldots, n)$. We must suppose $v \in S_{i i}$ since $f^{\prime}$ is a colouring of $C^{\prime}$. The compound $R=\left\{P_{1}, P_{2}, \ldots, P_{n}\right\}_{v}$ is non-trivial. It is therefore preceded by a basic colour-proposition $R^{\prime}=\left[Z_{1}, Z_{2}, \ldots, Z_{n}\right]$ of $C$ of rank $\leq 2$. (Possibly $R^{\prime}=R$.) But then the above definitions imply that $R^{\prime}$ is a basic colour-proposition of $C^{\prime}$ and that $f^{\prime}(x)=i$ for each $x \in Z_{i}(i=1,2, \ldots, n)$. This contradicts the definition of $f^{\prime}$ as a colouring of $\mathrm{C}^{\prime}$. The theorem follows.

Returning to the graph $G$ we observe that it is permissible to assert of the null subset $\emptyset$ of $V(G)$ that every member of $\emptyset$ has the colour $i(i=1,2, \ldots, n)$. So the statement that $G$ has no $n$-colouring is equivalent to the assertion that $[\emptyset, \emptyset, \ldots, \emptyset]$ is a colour-proposition. Applying theorem 1 we obtain

THEOREM 2. G has no n-colouring if and only if $[\emptyset, \emptyset, \ldots, \emptyset]$ is a member of the hierarchy $H(G)$.

University of Toronto 